\title{
Sexual dysfunction in Huntington's Disease: what do we really know?
}

\author{
Philip W. Tipton \\ Department of Neurology, Mayo Clinic, Jacksonville, Florida, United States
}

\begin{abstract}
Introduction. In this edition, Szymuś et al. conducted a systematic review revealing sexual dysfunction to be more prevalent in patients with Huntington's Disease compared to controls.

Clinical reflections. Sexual dysfunction in HD (SDHD) is common and significantly affects patient quality of life. Commonly used HD rating scales and treatment guidelines do not explicitly address SDHD, and research studies are limited by size and methodology.

Clinical implications. It is important that validated sexual dysfunction screening tools be utilised in clinical and research settings.
\end{abstract}

Key words: Huntington's Disease, sexual dysfunction

(Neurol Neurochir Pol 2020; 54 (4): 291-293)

Huntington's Disease (HD) is a dominantly inherited neurodegenerative disease caused by an expanded CAG trinucleotide repeat in the HTT gene, which encodes the huntingtin protein [1]. Symptoms of HD include a classic triad of movement abnormalities, cognitive impairment, and behavioural disturbances. Due to genetic anticipation, these symptoms occur earlier, and more severely, with successive generations [2]. HD affects people during the prime years of life with an average age at onset of between 30 and 50 [3]. While HD is rare, affecting only 2.71 per 100,000 people worldwide [4], associated symptoms cause significant functional impairment [5]. HD is the most common cause of inherited chorea, and clinicians may focus on the movement disorder at the expense of other symptoms that cause as much, if not more, detriment to quality of life (QoL).

Szymuś et al. conducted a systematic review of HD-associated sexual dysfunction [6]. They found that the majority of patients with HD had sexual disorders, particularly hypo/ hyperactive sexual disorder, erectile and ejaculatory dysfunction, lubrication problems, and orgasmic dysfunction. Their findings suggest that $\mathrm{SD}$ is more common in $\mathrm{HD}$ and correlates with QoL measures [6]. However, their review also illuminates several limitations of the current literature regarding SDHD.
Firstly, the HD literature has consistently underinvestigated sexual function. The DSM-5 has an entire chapter on SD, which includes delayed ejaculation, erectile disorder, female orgasmic disorder, female sexual interest/arousal disorder, genito-pelvic pain/penetration disorder, male hypoactive sexual desire disorder, premature ejaculation, substance/ medication-induced SD, and others [7]. This categorisation alone illustrates the variety of elements to consider when evaluating patients for SD. However, widely used HD rating scales e.g. UHDRS and UHDRS-FAP, and current treatment guidelines, do not address SD at all [8-11].

Therefore, it is not surprising that SDHD has been overshadowed by other disease symptoms such as chorea. Moreover, conducting a thorough assessment of sexual function can be time-consuming for clinicians and awkward or uncomfortable for patients and their partners. This method of verbal assessment can lead to underreporting of sexual dysfunction for a variety of reasons relating to one's culture, belief set, or social factors. Validated sexual function scales can help with this problem. The international index of erectile function (IIEF) assesses erectile function, orgasmic function, sexual desire and intercourse satisfaction in males [12]. For females, the female sexual function index (FSFI) yields a valid assessment of sexual domains including desire, arousal, lubrication, orgasm,

Address for correspondence: Philip W. Tipton, Department of Neurology, Mayo Clinic, 4500 San Pablo Rd., Jacksonville, FL 32224, USA,

e-mail: tipton.philip@mayo.edu 
satisfaction and pain [13]. Validated scales such as the IIEF and FSFI should be the standard for future studies.

Another limitation of the literature is a simple lack of well-designed, fully-powered studies focusing on SDHD as a primary endpoint. Szymuś et al. found only nine studies conducted over a 25-year timespan. These included information on sexual function of at least 20 genetically confirmed HD patients [6]. These studies were conducted an average of 11 years ago, and one third were conducted more than 18 years ago. There also seems to be an inverse relationship between study size and data quality. The largest study, of more than 2,500 patients, used unvalidated online survey questions with no control group [14]. Only two small trials used validated sexual function scales i.e. the IIEF [15] and the FSFI [16]. Non-standardised study methodology, and an apparent trade-off between study size and quality, inhibit us from developing reliable conclusions.

A recent study found that SDHD resulted in the highest life impact among $216 \mathrm{HD}$-related symptoms [5]. Despite this substantial effect on QoL, we know little about the mechanisms underlying SDHD. It is unclear whether the SD is directly caused by the HD pathomechanism, or whether it is merely a byproduct of other symptoms and/or their treatments. Depression is prevalent in HD [17] and is directly related to erectile/ejaculatory problems [18]. Dopamine depleting agents such as tetrabenazine are effective and widely used to treat HD-related chorea [19]; however, this mechanism can also cause depressive symptoms or exacerbate preexisting depression [20]. There is also a well-established association between antidepressants and SD [21]. These are just some of the factors that are likely to be contributory to SDHD.

This is an exciting time for HD research, with the development of enhanced rehabilitative strategies and promising disease-modifying treatments such as antisense oligonucleotides (ASO) [22-24]. It remains to be seen how these interventions will affect patients at different disease stages. Further research is required to study large patient groups using validated assessment tools. Lastly, clinicians should remember that SDHD substantially affects QoL, and that patients frequently withhold such information due to a variety of cultural and societal factors.

\section{Funding sources for this study: None}

Financial disclosure/conflict of interest:

Dr. Tipton reports no disclosures or conflicts of interest

\section{References}

1. Gusella JF, Wexler NS, Conneally PM, et al. A polymorphic DNA marker genetically linked to Huntington's disease. Nature. 1983; 306(5940): 234-238, doi: 10.1038/306234a0, indexed in Pubmed: 6316146.

2. Kieburtz K, MacDonald M, Shih C, et al. Trinucleotide repeat length and progression of illness in Huntington's disease. J Med Genet.
1994; 31(11): 872-874, doi: 10.1136/jmg.31.11.872, indexed in Pubmed: 7853373.

3. Roos RAC. Huntington's disease: a clinical review. Orphanet J Rare Dis. 2010; 5: 40, doi: 10.1186/1750-1172-5-40, indexed in Pubmed: 21171977.

4. Pringsheim T, Wiltshire $\mathrm{K}$, Day $\mathrm{L}$, et al. The incidence and prevalence of Huntington's disease: a systematic review and meta-analysis. Mov Disord. 2012; 27(9): 1083-1091, doi: 10.1002/mds.25075, indexed in Pubmed: 22692795.

5. Glidden AM, Luebbe EA, Elson MJ, et al. Patient-reported impact of symptoms in Huntington disease: PRISM-HD. Neurology. 2020 [Epub ahead of print], doi: 10.1212/WNL.0000000000008906, indexed in Pubmed: 32193209.

6. Szymuś K, Bystrzyński A, Kwaśniak-Butowska M, et al. Sexual dysfunction in Huntington's Disease - a systematic review. Neurol Neurochir Pol. 2020 [Epub ahead of print], doi: 10.5603/JNNS.a2020.0025, indexed in Pubmed: 32242915.

7. American Psychiatric Association Diagnostic and Statistical Manual of Mental Disorders (DSM-IV). SpringerReference. , doi: 10.1007/ springerreference_179660.

8. Unified Huntington's Disease Rating Scale: reliability and consistency. Huntington Study Group. Mov Disord. 1996; 11(2): 136-142, doi: 10.1002/mds.870110204, indexed in Pubmed: 8684382.

9. Winder JY, Achterberg WP, Marinus J, et al. Assessment Scales for Patients with Advanced Huntington's Disease: Comparison of the UHDRS and UHDRS-FAP. Mov Disord Clin Pract. 2018; 5(5): 527-533, doi: 10.1002/mdc3.12646, indexed in Pubmed: 30515443.

10. Youssov K, Dolbeau G, Maison P, et al. The unified Huntington's Disease Rating Scale for advanced patients: validation and follow-up study. Mov Disord. 2013; 28(14): 1995-2001, doi: 10.1002/ mds.25678, indexed in Pubmed: 24123464.

11. Armstrong MJ, Miyasaki JM. Evidence-Based Guideline: Pharmacologic Treatment of Chorea in Huntington Disease. 2012. www.neurology. org (27.03.2020).

12. Rosen RC, Riley A, Wagner $G$, et al. The international index of erectile function (IIEF): a multidimensional scale for assessment of erectile dysfunction. Urology. 1997; 49(6): 822-830, doi: 10.1016/s00904295(97)00238-0, indexed in Pubmed: 9187685.

13. Rosen R, Brown C, Heiman J, et al. The Female Sexual Function Index (FSFI): a multidimensional self-report instrument for the assessment of female sexual function. J Sex Marital Ther. 2000; 26(2): 191-208, doi: 10.1080/009262300278597, indexed in Pubmed: 10782451.

14. Simpson JA, Lovecky D, Kogan J, et al. Survey of the Huntington's Disease Patient and Caregiver Community Reveals Most Impactful Symptoms and Treatment Needs. J Huntingtons Dis. 2016; 5(4): 395-403, doi: 10.3233/JHD-160228, indexed in Pubmed: 27983566.

15. Kolenc M, Kobal J, Podnar S. Male sexual function in presymptomatic gene carriers and patients with Huntington's disease. J Neurol Sci. 2015; 359(1-2): 312-317, doi: 10.1016/j.jns.2015.11.015.

16. Kolenc M, Kobal J, Podnar S. Female Sexual Dysfunction in Presymptomatic Mutation Carriers and Patients with Huntington's Disease. J Huntingtons Dis. 2017; 6(2): 105-113, doi: 10.3233/jhd-160224.

17. Aldaz T, Nigro P, Sánchez-Gómez A, et al. Non-motor symptoms in Huntington's disease: a comparative study with Parkinson's disease. J Neurol. 2019; 266(6): 1340-1350, doi: 10.1007/s00415-01909263-7, indexed in Pubmed: 30834978.

18. Aziz NA, Anguelova GV, Marinus J, et al. Autonomic symptoms in patients and pre-manifest mutation carriers of Huntington's disease. 
Eur J Neurol. 2010; 17(8): 1068-1074, doi: 10.1111/j.1468-1331.2010.02973.x, indexed in Pubmed: 20192977.

19. Jankovic J. Dopamine depleters in the treatment of hyperkinetic movement disorders. Expert Opin Pharmacother. 2016; 17(18): 2461-2470, doi: 10.1080/14656566.2016.1258063, indexed in Pubmed: 27819145.

20. Kenney C, Hunter C, Jankovic J. Long-term tolerability of tetrabenazine in the treatment of hyperkinetic movement disorders. Mov Disord. 2007; 22(2): 193-197, doi: 10.1002/mds.21222, indexed in Pubmed: 17133512.

21. Gregorian RS, Golden KA, Bahce A, et al. Antidepressant-induced sexual dysfunction. Ann Pharmacother. 2002; 36(10): 1577-1589, doi: 10.1345/aph.1A195, indexed in Pubmed: 12243609.
22. Mirek E, Filip M, Chwała W, et al. The influence of motor ability rehabilitation on temporal-spatial parameters of gait in Huntington's disease patients on the basis of a three-dimensional motion analysis system: An experimental trial. Neurol Neurochir Pol. 2018; 52(5): 575-580, doi: 10.1016/j.pjnns.2018.02.001, indexed in Pubmed: 29475565.

23. Leavitt B, Kordasiewicz H, Schobel S. Huntingtin-Lowering Therapies for Huntington Disease. JAMA. 2020, doi: 10.1001/jamaneurol.2020.0299.

24. Tabrizi SJ, Leavitt BR, Landwehrmeyer GB, et al. Phase 1-2a IONIS-HTTRx Study Site Teams. Targeting Huntingtin Expression in Patients with Huntington's Disease. N Engl J Med. 2019; 380(24): 2307-2316, doi: 10.1056/NEJMoa1900907, indexed in Pubmed: 31059641. 